\title{
PULSED ULTRAVIOLET LIGHT DECONTAMINATION OF ARTIFICALLY-GENERATED MICROBIOLOGICAL AEROSOLS *
}

\author{
L. R. Dougall ${ }^{\xi}$ 1), J. B. Gillespie 1), M. Maclean ${ }^{1,2)}$, I. V. Timoshkin ${ }^{1)}$, M. P. Wilson'), S. J. \\ MacGregor1) \\ 1) The Robertson Trust Laboratory for Electronic Sterilisation Technologies, Department of \\ Electronic \& Electrical Engineering, University of Strathclyde, Royal College Building, 204 George \\ Street, Glasgow, G1 1XW, Scotland, UK \\ 2) Department of Biomedical Engineering, University of Strathclyde, Glasgow, UK
}

\section{Abstract}

Airborne transmission of infectious organisms is a major public health concern, particularly within healthcare and communal public environments. Methods of environmental decontamination utilising pulsed ultraviolet (UV) light are currently available, however it is important that germicidal efficacy against airborne contamination is established.

In this study bacterial aerosols were generated and exposed to short duration pulses $(\sim 20 \mu \mathrm{s})$ of UV-rich light emitted from a xenon-filled flashlamp. The lamp was operated using a $1 \mathrm{kV}$ solid-state pulsed power source, with a pulse frequency of $1 \mathrm{~Hz}$, and output energy of $20 \mathrm{~J} /$ pulse. Post-treatment, air samples were extracted from the chamber and the surviving fraction was enumerated using standard microbiological culture methods. Results demonstrate successful aerosol inactivation, with a $92.1 \%$ reduction achieved with only 5 pulses of UV-rich light $(\mathrm{P}=<0.0002)$. Inactivation using continuous UV light was also investigated in order to quantify the comparative efficacy of these antimicrobial light sources. Overall, results provide evidence of the comparative efficacy of pulsed and continuous UV light for inactivation of airborne bacterial contamination. For practical application, given the safety restrictions limiting its application for decontamination of unoccupied environments, or within sealed enclosures such as air handling units, the reduced treatment times with PUV provides significant operational advantages over continuous light treatment.

\section{INTRODUCTION}

Biological aerosols are a serious threat to human health due to their role in the transmission of infection and disease. This is particularly important in the hospital environment, as well as areas of public congregation such as schools and methods of public transport. Additionally, the threat of intentional or accidental release of bioaerosols as agents of bioterrorism exists. Extensive research has been conducted on sterilisation of potentially dangerous pathogens on surfaces and in liquids. In contrast however, there is a lack of evidence reflecting the efficacy of air sterilisation.

In hospitals, the spread of infection and disease via the airborne route is not fully understood and therefore, is often overlooked. However, airborne transmission is the major route of infection for a number of highly infectious diseases such as Mycobacterium tuberculosis, and has been implicated in the spread of many clinicallyimportant pathogens such as methicillin-resistant Staphylococcus aureus (MRSA). It is estimated that hospital-acquired infections cost the UK National Health Service (NHS) more than $£ 1$ billion a year, and the contribution of airborne transmission has been estimated to account for $10-33 \%$ [1-3]. Traditional approaches to disinfection involve manual cleaning with chemicals however, a study by Carling et al showed that as much as $50 \%$ of high touch surfaces within patient settings were missed [4].

In response, novel technologies are being developed to compliment manual cleaning, such as 'no touch' environmental disinfection systems. One such example is the use of ultraviolet (UV) irradiation, which has been investigated for surfaces as well as air disinfection, where manual cleaning is not practical. The antimicrobial properties of UV light are well established, specifically, UV-C light between $190-290 \mathrm{~nm}$ with a peak germicidal output at approximately $260 \mathrm{~nm}$. The mechanism by which UV-C radiation inactivates microorganisms involves damage to their deoxyribonucleic acid (DNA). Inactivation occurs when pyrimidine dimers form between adjacent thymine bases in response to absorption of a photon, which prevents the microbe from replicating, rendering it harmless [5].

Clinical applications of UV-C sterilisation have utilised continuous and pulsed UV light for whole room decontamination. Continuous UV (CUV) light makes use

\footnotetext{
* This work has been supported by an EPSRC doctoral training grant.

$\xi$ email: laura.dougall@strath.ac.uk
} 
of a low pressure mercury lamp at $254 \mathrm{~nm}$ or a medium pressure mercury lamp. Several commercially available CUV systems are undergoing clinical trial for use in the hospital environment. A study by Nerandzic et al demonstrated a $2-4 \log _{10}$ reduction of MRSA, C. difficile and VRE using a commercially available UV-C decontamination device [6]. However, CUV systems require application over long periods of time with low power output to generate significant inactivation. In contrast, pulsed UV (PUV) is a more desirable method of sterilisation, which uses pulsed polychromatic xenon flash lamps to allow energy to be applied over a short period of time with high peak power. Short pulses of high intensity light cause rapid inactivation of microorganisms and inactivation has been shown to be effective in liquid, on surfaces and in air. The main aim of this study was to establish the susceptibility of airborne bacterial contamination to PUV by establishing dose-response kinetics, and comparison of the germicidal efficacy to CUV light treatment.

\section{EXPERIMENTAL ARRANGEMENT}

\section{A. Microbiological aerosol preparation}

The bacterial strain used throughout this study was Staphylococcus epidermidis LMG 10273 (Laboratorium voor Microbologie, Universiteit Gent, Belgium). For experimental use, S. epidermidis was cultured in $100 \mathrm{~mL}$ Tryptone Soya Broth (Oxoid Ltd, UK) at $37^{\circ} \mathrm{C}$ under rotary conditions (120 rpm) for 18-24-h. Post incubation the bacterial suspension was centrifuged at $3939 \times \mathrm{g}$ for 10 minutes and the pellet re-suspended in $100 \mathrm{~mL}$ phosphate buffered saline (PBS) (Oxoid Ltd, UK), and serially diluted to obtain the required cell density (colony

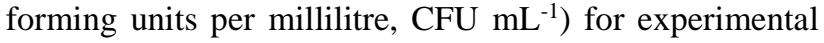
use. $60 \mathrm{~mL}$ of bacterial suspension at a starting population

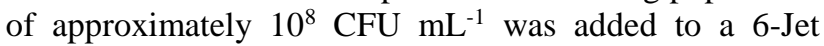
Collison nebuliser connected to the test chamber. Bacterial aerosols were generated by operating the nebuliser for 1 minute at $20 \mathrm{psi}$, giving a suspended population of approximately $6 \times 10^{8} \mathrm{CFU} \mathrm{mL}^{-1}$ in the chamber at the start of the exposure period.

\section{B. Experimental system}

The aerosol suspension chamber, shown in Figure 1, was a $15 \mathrm{~L}$ cylindrical acrylic tube with a diameter of 24 $\mathrm{cm}$ and height of $35 \mathrm{~cm}$. The chamber lid contained a quartz glass window with a $13 \mathrm{~cm}$ diameter, to permit light transmission. The UV light source was mounted above the quartz window and housed within an outer casing to prevent stray light exposure. An inlet for the nebuliser and outlet for the BioSampler were also present and could be sealed with HEPA vents during exposure. A small fan was attached to the base of the inside of the chamber to aid aerosol circulation. Bacterial aerosols were exposed to increasing durations of UV-light treatment.

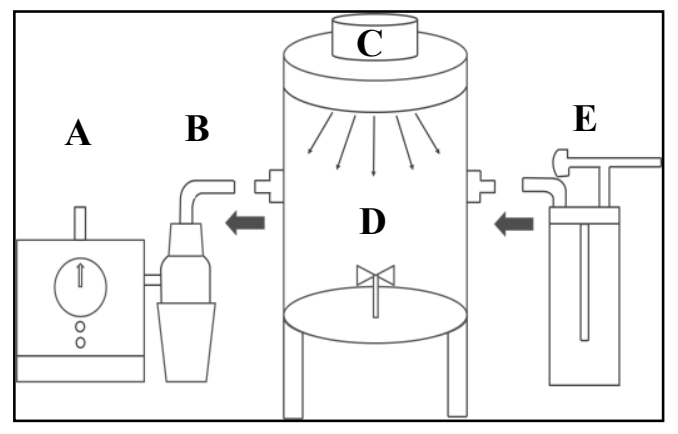

Figure 1. Schematic diagram of experimental set-up. $A=$ vacuum pump, $\mathrm{B}=$ BioSampler liquid impinger, $\mathrm{C}=$ light source, $\mathrm{D}=$ aerosol chamber and $\mathrm{E}=$ nebuliser.

\section{PUV and CUV light treatment}

A polychromatic low pressure $100 \mathrm{~W}$ xenon filled flash lamp was connected to a $1 \mathrm{kV}$ solid-state pulsed power generator (Samtech, UK) with a pulse frequency of 1 pps and output energy of $20 \mathrm{~J} /$ pulse. The capacitive discharge circuit is shown in Figure 2. Stored electrical energy was transferred from the solid-state power generator to the xenon filled flash lamp at 1 pulse/second, discharging short pulses of broadband light that ranged from UV to IR, with a high UV output required for microorganism inactivation. The short duration pulses of PUV-light have an exponentially decaying waveform, shown in Figure 3 with half time of approximately $20 \mu \mathrm{s}$. The emission spectra from the PUV lamp is shown in Figure 4.

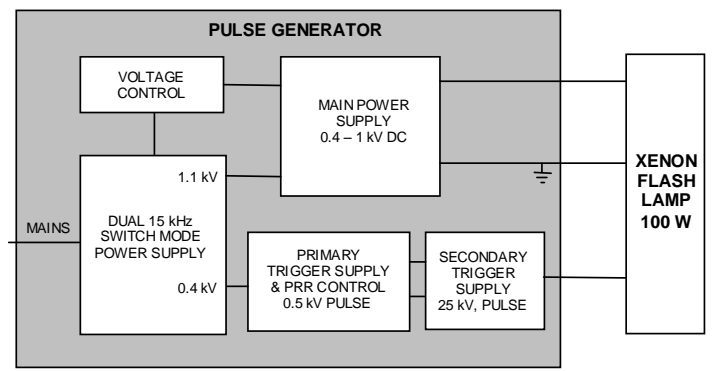

Figure 2. Components of the PUV generator and xenon flashlamp.

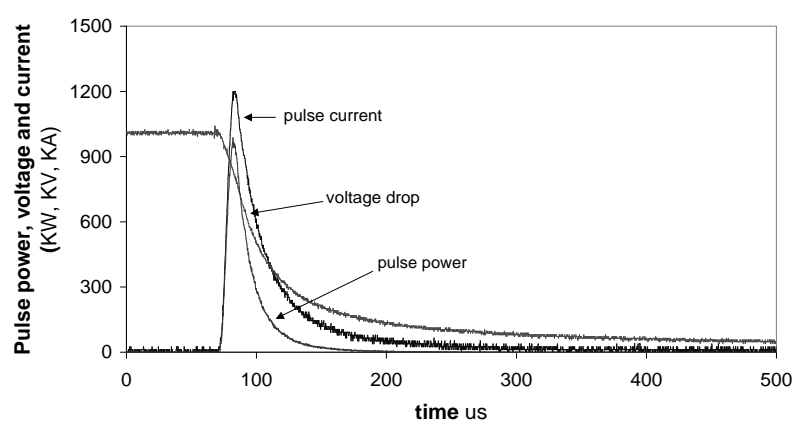

Figure 3. Waveforms of the xenon flashlamp. 


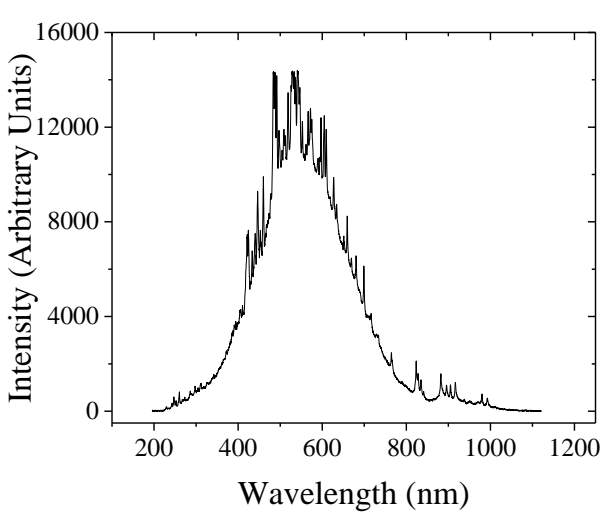

(a)

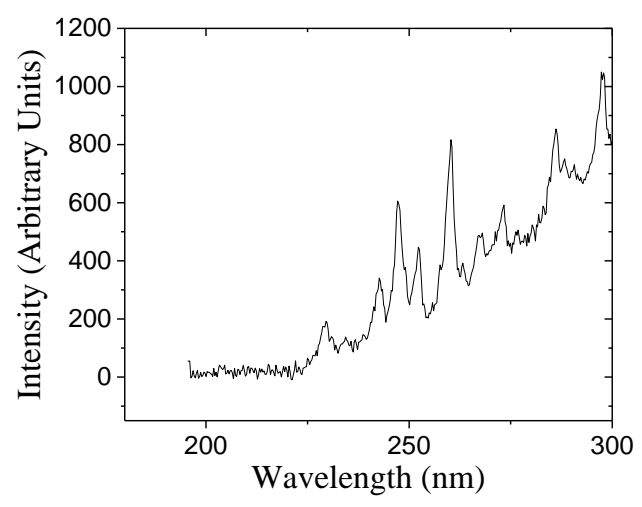

(b)

Figure 4. Optical emission spectra of the xenon flashlamp, measured using a HR4000 spectrometer (Ocean Optics, Germany). (a) total emission spectrum; (b) emission spectrum in UV-region.

The CUV source was a germicidal PLS 9w 2 pin G23 bulb (Easy LightBulbs, UK) which emits short wave radiation with a peak output at $253.4 \mathrm{~nm}$, as shown in Figure 5. Dose was calculated as irradiance $\left(\mathrm{mW} \mathrm{cm}^{-1}\right) \times$ exposure time (s) at an approximate irradiance of $900 \mu \mathrm{W}$ and $203 \mu \mathrm{W}$ for PUV and CUV respectively, measured at the maximum distance from the light source.

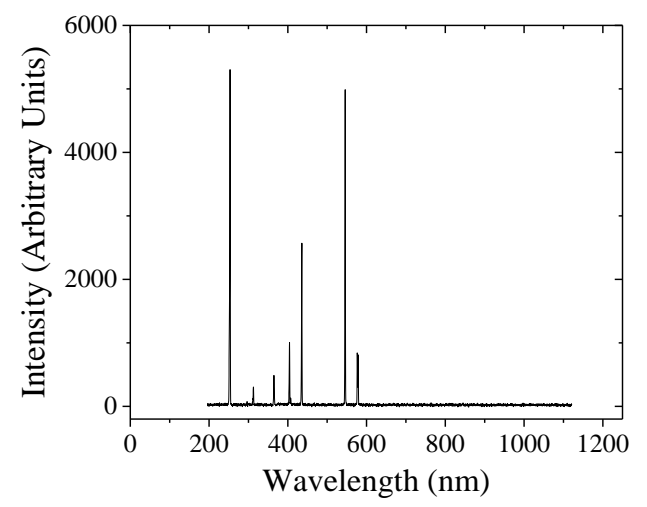

(a)

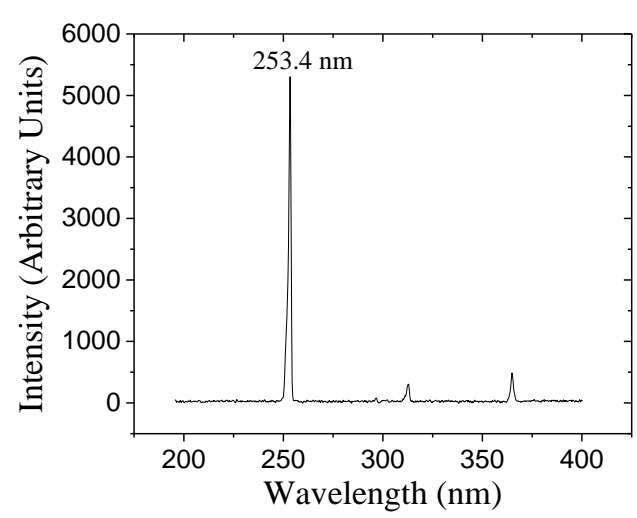

(b)

Figure 5. Optical emission spectra of the CUV-light source, measured using a HR4000 spectrometer (Ocean Optics, Germany). (a) total emission spectrum (b) emission spectrum in UV-region.

\section{Air sampling and enumeration}

Post-exposure, air samples were extracted from the test chamber using a BioSampler liquid impinger (SKC, UK). A vacuum pump connected to a glass collection vessel was operated at $12.5 \mathrm{~L} \mathrm{~min}^{-1}$ for 5 minutes to remove particle-laden air from the chamber and impact it into 20 $\mathrm{mL}$ of PBS held inside the collection vessel. Samples were serially diluted and plated in triplicate onto Tryptone Soya Agar (Oxoid Ltd, UK) using the Pour Plate Method and incubated at $37^{\circ} \mathrm{C}$ for 24-hrs. Samples were

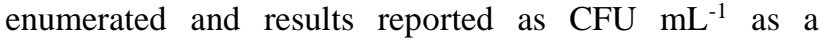
function of exposure time ( $\mathrm{min})$. Results are an average of a minimum of triplicate independent experiments, measured in triplicate $(n=9)$, with error bars representing the standard deviation (SD). Data were analysed using paired sample T-tests using Minitab Statistical software version 17, with significant differences identified at the $95 \%$ confidence interval, $\mathrm{P} \leq 0.05$.

\section{EXPERIMENTAL RESULTS AND DISCUSSION}

Results shown in Figure 6 demonstrate the effect of PUV-light exposure on airborne S. epidermidis. Significant reduction was achieved after 5 pulses of PUVlight at 1 pps. At this pulse number, a $92.1 \%$ reduction was observed when compared to the non-exposed control sample $(\mathrm{P}=0.0003)$. After 500 pulses of PUV-light at 1 pps, a $2.9 \log _{10}(99.8 \%)$ reduction was achieved, with $<1 \%$ of the starting population surviving at this dose $\left(\sim 450 \mu \mathrm{Jcm}^{-2}\right)$. These results support many studies published on rapid PUV-light inactivation of microorganisms. A commercially available PUV-light disinfection system resulted in a $5-\log _{10}$ reduction 


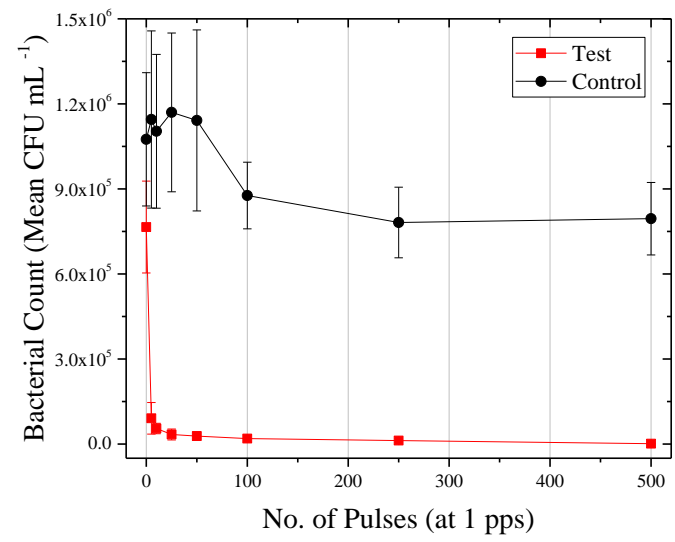

(a)

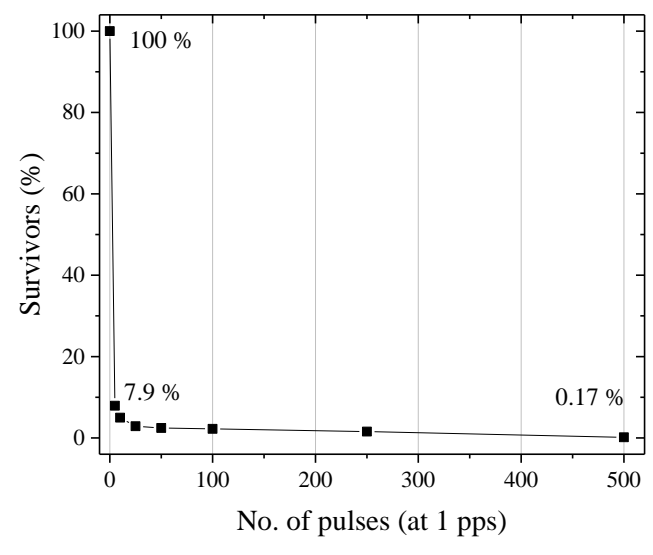

(b)

Figure 6. Susceptibility of aerosolised $S$. epidermidis to PUV-light. Optical irradiance was $900 \mu \mathrm{W}$ measured at a maximum distance of $35 \mathrm{~cm}$ from the light source. (a) Inactivation kinetics; (b) Percentage surviving population data $(\mathrm{n} \geq 9 \pm \mathrm{SD})$. * Significant inactivation $(\mathrm{P}<0.05)$

(99.999\%) of 4 different medically relevant pathogens in a UK hospital in just 10 minutes [7]. The same system was found to be $7 \times$ more effective than traditional cleaning and resulted in an $87 \%$ reduction in Intensive Care infection rates [8]. Of note, these studies do not directly analyse airborne disinfection, however it is highly likely both surface and airborne contamination were reduced.

However, a number of trials which have been carried out to compare the efficacy of PUV and CUV-light exposure found that bacterial reduction was not significantly higher with the PUV system, and one study found it to be lower than with CUV. Results shown in Figure 7 demonstrate the effect of CUV-light exposure on airborne S. epidermidis. Significant reduction was achieved after an initial 5 minute exposure of CUV-light. At this exposure time, a $98.7 \%$ reduction was observed when compared to the non-exposed control sample $(\mathrm{P}=0.00003)$.

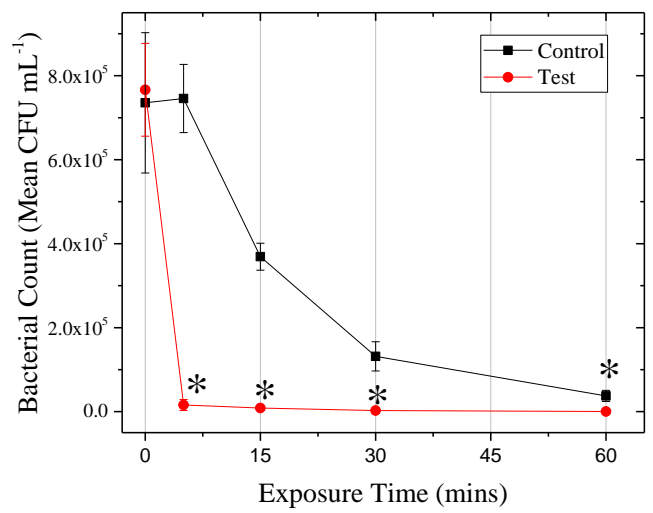

(a)

Figure 7. Susceptibility of aerosolised S. epidermidis to CUV-light. Optical irradiance was $203 \mu \mathrm{W}$ measured at a maximum distance of $35 \mathrm{~cm}$ from the light source. (a) Inactivation kinetics; (b) Percentage surviving population data $(\mathrm{n} \geq 9 \pm \mathrm{SD}) . *$ Significant inactivation $(\mathrm{P}<0.05)$

After 60 minutes light treatment, a 3.2 - $\log _{10}$ reduction was achieved, with $<2 \%$ of the starting population surviving at this dose $\left(\sim 730 \mu \mathrm{Jcm}^{-2}\right)$.

CUV-light offers operational advantages such as low running costs and less maintenance and has therefore been installed in many healthcare settings as a method of upper room germicidal air disinfection. As evidenced in the results of the present study, the time required to achieve a similar germicidal efficacy to that of PUV light is much longer, and this is a major disadvantage of CUV-light.

It is generally considered that bacterial inactivation kinetics in liquids do not predict the kinetics of microbial inactivation in air. King et al also found that organisms appear to me more susceptible to UV irradiation when suspended in the air [9]. This is thought to be due to a higher surface area exposure to UV irradiation when the organism is moving and rotating in the air, in contrast to being fixed onto a surface.

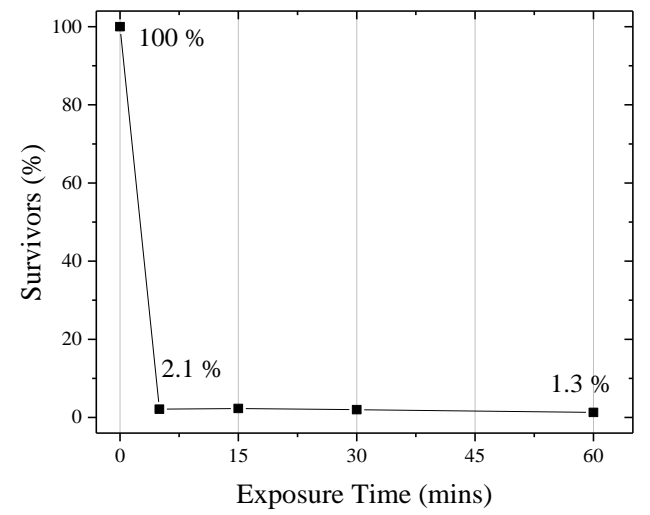

\section{(b)}


Although both PUV and CUV-light irradiation achieved $99.8 \%$ and $98.7 \%$ reduction respectively, complete bacterial inactivation was not achieved. Both decay curves ended in a tail, indicating a resistant population. A possible explanation for this phenomenon is bacterial clustering. If numerous bacteria clump together, those in the centre of the cluster will be shielded from UV-light exposure, and thus, appear to be resilient. Kesevan and Sagripanti stated that the linear section of an inactivation curve would stop at $3-\log _{10}$ if just 1 in 1000 bacteria was shielded. Similarly if one bacterium was shielded by 2 others, then only $37 \%$ of the irradiated light would reach that third bacterium [10].

Natural decay of the suspended aerosol was also observed in both experiments, and this was more prominent in the CUV-light curve where the aerosol required longer suspension times inside the test chamber. This is potentially attributable to a number of factors including natural gravitational settling, impaction with the chamber walls, shear stress in the nebuliser or nondesirable environmental conditions of temperature and relative humidity. King et al (2011) experienced a $10 \%$ loss of viable bacteria inside their test chamber after 170330 seconds.

\section{CONCLUSIONS}

This study has demonstrated evidence of the susceptibility of bacterial aerosols to both PUV and CUVlight. Dose-response kinetics of airborne S. epidermidis to PUV-light were established and compared to that of CUV-light. PUV light was found to be more efficient for decontamination of airborne bacteria due to rapid energy delivery with high peak power, in comparison to lower energy output of CUV light. The approximate maximum dose of PUV light was $450 \mathrm{~mW} \mathrm{~cm}^{-2}$ and $730.8 \mathrm{~mW} \mathrm{~cm}^{-2}$ for CUV-light. However, the CUV light had to be operated for $>7$ times longer than PUV light in order to achieve similar bacterial reduction (500 seconds and 3600 seconds for PUV and CUV, respectively).

Overall, both continuous and pulsed UV-C disinfection methods have a rapid decontamination effect with widespread antimicrobial efficacy against a range of healthcare-associated pathogens. However this light-based technology is restricted to terminal room cleaning and unoccupied environments due to the carcinogenic and mutagenic nature of UV-C light, therefore the reduced treatment times of PUV-light can provide significant operational advantages over CUV-light treatment.

\section{REFERENCES}

[1] P. S. Brachman, "Nosocomial infection - airborne or not? Proceedings of the International Conference on Nosocomial Infections", American Hospital Association, 1970, pp. 189-192.

[2] W. J. Kowalski, "Air treatment systems for controlling hospital acquired infections", HPAC Engineering, vol. 79 (no. 1), pp. 28-48, 2007.

[3] House of Commons Public Accounts Committee. "Reducing healthcare-associated infections in hospitals in England", Fifty-second report of session 2008-9. [Online] Available:http://www.publications.parliment.uk/pa/cm200 809/cmselect/cmpubacc/812/812.pdf. Accessed 15/07/17.

[4] P. C. Carling, J. L. Briggs, J. Perkins, D. Highlander, "Improved cleaning of patient rooms using a new targeted method", Clin. Infect. Dis., vol. 42, pp. 385-388, 2006.

[5] N. G. Reed, "The history of ultraviolet germicidal irradiation for air disinfection", Public. Health. Rep., vol. 125, pp. 15-27, 2010.

[6] M. Nerandzic, J. L. Cadnum, M. J. Pultz, C. J. Donskey, "Evaluation of an automated ultraviolet radiation device for decontamination of Clostridium difficile and other healthcare-associated pathogens in hospital rooms", BMC Infect. Dis., vol. 10, pp. 197, 2010.

[7] I. Hosein, R. Madeloso, W. Nagaratnam, F. Villamaria, E. Stock, C. Jinadatha, "Evaluation of a pulsed xenon ultraviolet light device for isolation room disinfection in a United Kingdom hospital", Am. J. Infect. Control., vol. 44 (no. 9), pp. e157-161, 2016.

[8] P. G. Vianna, C. R. Dale Jr, S. Simmons, M. Stibich, C. M. Licitra, "Impact of pulsed xenon ultraviolet light on hospital-acquired infection rates in a community hospital", Am. J. Infect. Control., vol. 44 (no. 3), pp. 299303, 2016.

[9] B. King, J. Kesavan, J. L. Sagripanti, "UV sensitivity of bacteria in aerosols and on contaminated surfaces", Aerosol Sci. Technol., vol. 45, pp. 645-653, 2011.

[10] J. Kesavan, J. L. Sagripanti, "Disinfection of airborne organisms by ultraviolet C radiation and sunlight", in Aerosol Science: Technology and Applications, I. Colbeck, M. Lazaridis, $1^{\text {st }}$ Ed., West Sussex: John Wiley and Sons Ltd, 2012, pp. 417-439. 